\title{
Globe
}

Revue internationale d'études québécoises

\section{Jean-Marc Larouche et Guy Ménard [éd.]: L'étude de la religion au Québec. Bilan et prospective. Québec, Presses de l'Université Laval et la Corporation canadienne des sciences religieuses, 2001}

\section{Lucia Ferretti}

Volume 6, numéro 2, 2003

URI : https://id.erudit.org/iderudit/1000824ar

DOI : https://doi.org/10.7202/1000824ar

Aller au sommaire du numéro

Éditeur(s)

Globe, Revue internationale d'études québécoises

ISSN

1481-5869 (imprimé)

1923-8231 (numérique)

Découvrir la revue

Citer ce compte rendu

Ferretti, L. (2003). Compte rendu de [Jean-Marc Larouche et Guy Ménard [éd.]: L'étude de la religion au Québec. Bilan et prospective. Québec, Presses de

l'Université Laval et la Corporation canadienne des sciences religieuses, 2001].

Globe, 6(2), 182-186. https://doi.org/10.7202/1000824ar d'utilisation que vous pouvez consulter en ligne.

https://apropos.erudit.org/fr/usagers/politique-dutilisation/ 


\title{
Jean-Marc Larouche et Guy Ménard [éd.]
}

\author{
L'étude de la religion au Québec. \\ Bilan et prospective. \\ Québec, Presses de l'Université Laval \\ et la Corporation canadienne des sciences \\ religieuses, 2001.
}

Est-ce seulement, comme l'affirment Larouche et Ménard en présentation, que l'arrivée d'un nouveau millénaire a nourri le désir d'une pause réflexive au milieu de la course effrénée qu'est devenue la vie universitaire? Dans sa préface, Louis Rousseau exprime ce que ressentent tous ses collègues, à savoir que la recherche sur la religion, celle de type théologique ou religiologique tout au moins, s'effectue au Québec dans un contexte adverse. Non seulement l'intelligentsia et la société globale ont rejeté le catholicisme depuis une quarantaine d'années et dénigré les Églises et les fois jusqu'à ne plus pouvoir comprendre, dans l'ensemble, l'expérience religieuse ; mais s'est ajoutée à cela, depuis près de dix ans, la menace très réelle de fermeture que font peser sur les départements de théologie ou de sciences religieuses des administrations universitaires tributaires elles aussi des préjugés collectifs et peu au courant de la recherche qui s'y fait. Nul doute, donc, que ce livre est né aussi du désir des spécialistes des études religieuses qui travaillent dans ces départements de faire connaître la valeur des traditions de la recherche québécoise ainsi que les travaux qu'ils mènent actuellement. Habités par ce sentiment commun d'une certaine urgence de dire et guidés par la commande très claire (bilan et prospective) que leur avaient passée les directeurs de l'ouvrage, la plupart des collaborateurs ont réellement donné le meilleur d'eux-mêmes, ce qui fait de ce livre une très belle réussite.

Une très belle réussite, à condition toutefois de prendre le livre pour ce qu'il est et non pour ce qu'il annonce. Dans un ouvrage intitulé L'étude de la religion au Québec, on s'attend légitimement à ce que tous les chercheurs qui font avancer le savoir sur la religion trouvent une place, quels que soient leurs horizons disciplinaires. Or, ce n'est pas le cas, loin de là, puisque le livre n'est centré que sur ce qui s'est fait, se fait et pourrait se faire dans les départements de théologie ou de sciences religieuses. Cependant, une fois qu'on a compris que le titre déborde le 
contenu réel du livre, on est prêt à découvrir l'éventail des préoccupations de recherche des collègues théologiens ou religiologues.

Pour appréhender ce vaste champ, Larouche et Ménard ont choisi quatre entrées : les traditions religieuses de l'humanité, les textes sacrés et les traditions religieuses anciennes, les nouvelles manifestations du religieux dans la culture, et enfin les liens entre religion, pratiques sociales et productions culturelles. Ce découpage, explicitement retenu parmi d'autres possibles, traduit à la fois les préoccupations des deux directeurs et les partis pris heuristiques dominants des études sur la religion menées dans les départements de théologie et de sciences religieuses depuis trente ans. Larouche et Ménard sont d'une part soucieux de donner une visibilité à tous les secteurs de la recherche, ceux qui s'appuient sur une tradition québécoise vieille déjà de plusieurs décennies autant que ceux qui en sont ici à leurs premiers balbutiements. Sans doute a-t-on vite fait le tour des travaux québécois sur le bouddhisme ou sur l'islam, mais au contraire, quelle richesse que notre tradition de recherche sur la Bible hébraïque ou le Nouveau Testament ! Tous ces secteurs bénéficient pourtant d'articles sensiblement de même longueur. Les directeurs reprennent aussi à leur compte cette idée généralement admise parmi nos collègues de ces départements d'un " déplacement " contemporain du religieux, et par conséquent de cette partie de la recherche qui le suit dans les " sectes ", le " nouvel âge ", et jusque dans plusieurs ritualités actuelles que des chercheurs d'autres horizons appréhenderaient comme tout à fait séculières. Larouche et Ménard, enfin, affirment tranquillement la position selon laquelle la religion, loin d'être un des modes possibles de présence au monde et de lecture de celui-ci, les traverse tous au contraire, si bien que la recherche en sciences religieuses et en théologie peut à son tour, légitimement, partir à la rencontre de toutes les pratiques sociales (langage, éthique ou sexualité) et de toutes les productions culturelles (arts, écologie, sciences humaines, par exemple). Tous ces choix éditoriaux entraînent des conséquences sur l'organisation du livre. Les directeurs eux-mêmes, tout comme Louis Rousseau ou Michel Despland, le postfacier, déplorent la sousreprésentation manifeste de l'abondante recherche sur le catholicisme et l'absence involontaire d'un article "Religion et politique ", certainement les deux principales lacunes de l'ouvrage, ou encore l'absence d'un article sur les études patristiques. On pourra aussi s'étonner de n'y pas trouver d'article " Religion et musique * par exemple, ou juger (parfois même en compagnie du collaborateur assigné) que certaines alliances 
ont été un peu forcées. À voir où est situé l'article sur "Les femmes, le féminisme et la religion ", on pourra enfin se questionner sur la relativité apparente accordée par les directeurs à la remise en question fondamentale apportée par le féminisme sur la manière de vivre et de penser la foi, la religion et l'Église. Mais ces quelques réserves n'ont pas altéré mon plaisir de constater la très grande variété des objets de recherche sur lesquels se penchent nos collègues théologiens et spécialistes des sciences religieuses, et la non moins grande diversité des perspectives dans lesquelles ils les abordent. De ce point de vue, le choix des angles d'approche privilégiés par Ménard et Larouche apparaît fort judicieux.

Aux collaborateurs, on demandait à la fois un bilan et une prospective. Les directeurs se sont souvent tournés vers des pionniers ou des piliers du secteur à couvrir. Richard Lougheed, par exemple, signe l'article sur " les traditions protestantes " et Frédéric Laugrand, celui sur "les traditions inuites $n$. Tous deux retracent méthodiquement l'histoire de la recherche dans ces secteurs et indiquent fort clairement les tendances actuelles. Parfois, au contraire, les éditeurs ont confié les dossiers à des jeunes, eux-mêmes en train de creuser de nouveaux sillons: Ėve Paquette sur "Religion et littérature " ou Robert Verreault sur les traditions amérindiennes. Certains collaborateurs, rares heureusement, se sont contentés d'énumérer et de résumer brièvement les travaux repérés dans leur domaine; cela affecte plus particulièrement quelques articles de la première partie. Tous les autres ont fait preuve d'un véritable effort intellectuel, s'astreignant à baliser le voyage qu'ils nous proposent dans l'histoire de leur champ de recherche, à dresser des typologies, à y classer les travaux, à faire voir comment se constituent, s'enracinent, se développent et vivent, de travaux individuels en travaux individuels, un savoir collectif et une communauté scientifique. J'ai senti beaucoup d'honnêteté dans ces contributions et une véritable capacité des auteurs de dépasser leurs intérêts individuels de recherche, leurs amitiés et inimitiés professionnelles en vue de présenter un panorama juste du secteur qui leur avait été confié. Sous ce rapport, Roger Marcaurelle est allé plus loin que les autres, puisqu'il a su indiquer aussi les lacunes et les points faibles des études dans les domaines qu'il couvre, soit ceux de la mystique et de la psychologie. En même temps, tous les collaborateurs se sont aussi pliés à l'exigence de la prospective : de cette façon, que de nouveaux objets de recherche s'offrent à la curiosité, à la patience et à l'intelligence des étudiants ! Un seul auteur, à mon sens, nous a donné encore davantage, soit un véritable essai : Robert Mager, sur "La 
transmission de la religion . Ici, Mager ne présente pas un portrait, il pose un problème ; les auteurs et leurs travaux ne sont pas à proprement parler l'objet de son texte, ils sont le support sur lequel il s'appuie pour établir sa propre perspective, construire sa propre réflexion. Un des sommets de l'ouvrage !

Me voici à parler maintenant du livre selon la perspective des lecteurs. De la lectrice que je suis, plutôt. J'hésite, par crainte d'injustice à l'égard des auteurs et des textes dont je ne dirai rien, mais qui, sous la plume d'un autre recenseur, trouveraient ou trouveront un écho mérité. Mais bon, il faut bien tout de même que je me commette, que je dise quelques-unes de mes si nombreuses découvertes, que je partage mes délices et mes souhaits. Allons-y un peu pêle-mêle. D'abord mes découvertes. Il est des domaines de recherche où le Québec fait figure de pionnier dans le monde scientifique francophone; je le savais pour la religion populaire, je l'ignorais pour la religiologie ou pour la recherche en éthique. J'ai découvert aussi que si les universitaires québécois de langue française ont commencé plus tard que leurs collègues anglophones à fouiller d'autres traditions religieuses que le catholicisme ou que les sources anciennes du christianisme, en revanche, il ne semble pas que les universitaires canadiens-anglais des départements de théologie ou de sciences religieuses de nos universités s'intéressent au catholicisme des Québécois francophones. J'ai appris encore, entre mille autres choses, que l'architecture religieuse est encore peu explorée dans la perspective des sciences religieuses, qui suppose, comme le dit Vicki Bennett, que les chercheurs tiennent compte des discours d'une communauté de fidèles sur son lieu de culte et sur ses devoirs envers Dieu, à qui il s'agit de construire un temple. Quelques délices maintenant : toute la seconde partie de l'ouvrage qui porte sur les textes sacrés et les traditions religieuses anciennes. Si l'on excepte les études sur notre milieu, c'est dans ces vastes et profonds domaines que la recherche québécoise, depuis des décennies, s'est fait un nom qui compte sur la scène internationale. La Bible hébraïque, le Nouveau Testament, la bibliothèque copte de Nag Hammadi, les traditions religieuses de l'Égypte et du Proche-Orient ancien, et les traditions de l'Occident médiéval : nous touchons là aux fondements mêmes de notre civilisation, et pour tous ces objets de recherche la contribution québécoise a été essentielle. Comment se fait-il que nous ne le sachions pas davantage ? Comment se fait-il que nos universités, si soucieuses de rayonnement, mesurent de plus en plus chichement le soutien à des équipes constituées de longue 
date, insérées organiquement dans les réseaux internationaux, et qui travaillent dans des champs dont, hors l'Université, aucune institution ne peut se charger? À l'heure où le monde s'est ému devant les événements tragiques que sont le saccage des grands bouddhas d'Afghanistan et le pillage des musées d'Irak, conservatoires de l'héritage mésopotamien, qu'il me soit permis d'exprimer le souhait que nous sachions mieux sensibiliser le grand public à ces entreprises fondamentales que mènent les équipes de ces secteurs ; peut-être alors nos universités s'apercevrontelles qu'elles ne doivent plus menacer ces trésors.

Dans une postface qui se lit comme un poème, Michel Despland se demande : "L'étude de la religion au Québec, comment? Pourquoi ? Comme on entreprend un voyage, répond-il, et pour les mêmes raisons, rencontrer l'autre et l'ailleurs, rester soi-même tout en s'ouvrant à l'universel. Chers collègues, merci pour ce beau livre.

Lucia Ferretti

Centre interuniversitaire d'études québécoises

Université du Québec à Trois-Rivières

\section{Mary Jean Green}

Women \& Narrative Identity.

Rewriting the Quebec National Text.

Montréal, McGill-Queen's University Press, 2001.

Dans l'ouvrage Women \& Narrative Identity, Mary Jean Green retrace le parcours de l'écriture des femmes au Québec, depuis le XIX ${ }^{e}$ siècle jusqu'à aujourd'hui. Plus précisément, il s'agit pour l'auteure de procéder à " une relecture féministe de la tradition littéraire au Québec ". Employé dans le sens où l'entend Adrienne Rich lorsqu'elle parle de revision, le terme " relecture "constitue une notion clé au plan méthodologique dans la mesure où Green entreprend de "revisiter" le roman identitaire des femmes afin de cerner leur contribution au développement de la culture québécoise. Dans l'introduction, l'auteure avance l'hypothèse que le genre romanesque au Québec a joué un rôle capital dans la construction d'une identité nationale et que les femmes ont élaboré, dans un contexte idéologiquement marqué par la survivance de la race canadiennefrançaise, depuis la fin du XIX $x^{e}$ siècle jusqu'à la Révolution tranquille, des 